\title{
Recent Techniques in Management of Lumbosacral Region Tumours: A Prospective Comparative Study
}

\author{
Mostafa Abd Elsamea, Mohamed Shaaban, Ahmed Taha* \\ Department of Neurosurgery, Al-Azhar University Hospital, Damietta, Egypt \\ Email: *ahmdth231@gmail.com
}

How to cite this paper: Elsamea, M.A., Shaaban, M. and Taha, A. (2018) Recent Techniques in Management of Lumbosacral Region Tumours: A Prospective Comparative Study. Open Journal of Modern Neurosurgery, 8, 239-249.

https://doi.org/10.4236/ojmn.2018.82020

Received: March 27, 2018

Accepted: April 24, 2018

Published: April 27, 2018

Copyright (c) 2018 by authors and Scientific Research Publishing Inc. This work is licensed under the Creative Commons Attribution International License (CC BY 4.0).

http://creativecommons.org/licenses/by/4.0/

\begin{abstract}
Background: The lumbosacral plexus consists of the ventral rami from the L2 to $\mathrm{S} 3$ nerve roots, with some additions from the $\mathrm{L} 1$ and $\mathrm{S} 4$ nerve roots as well. The standard treatment for spinal cord compression is urgent surgically decompressive laminectomy over the affected level with resection as much as possible of the exposed tumor to relieve the cord compression. We aim to correlate the impact of preoperative clinical picture, duration of symptoms, and the pathological nature of the lumbo-sacral spine tumors on the outcome of comparative study different modalities of management. Patients and Methods: During the period between 2015 and 2017, thirty cases were studied and surgically managed in Al-Azhar University Hospital, Damietta. Cases were selected for surgery if they were diagnosed as having a symptomatizing lumbosacral lesion. Results: Thirty patients were included in the current study; the commonest lesion in this study was ependymoma eight patients (27\%), astrocytoma six patients (20\%), and meningioma five patients (17\%). Nerve sheath tumor comprised five cases (17\%) with the hemangioblastomas three cases (10\%), epidermoid one case (3\%), and ependymoma of the filum terminale two cases (6\%). The mean age of the included patients was $38.96 \pm$ 15.42. The mean duration of lesions was $13.66 \pm 10.93$. They were 15 male patients (50\%), and 15 female patients (50\%), with male: female ratio 1:1. The mean duration of symptoms ranged for 1:60 months. The most common presenting manifestations were sensory followed by motor and sphinctric disturbance. Complications occurred in 8 patients (23\%), and the most common was postoperative CSF leak. 19 out of 30 cases were surgically totally resected (63\%), while 11 cases (37\%) were grossly totally resected. Conclusions: Ependymoma and meningioma have more favorable outcome compared to other spinal tumors. Accurate and rapid diagnosis prior to surgery also associated with good outcome.
\end{abstract}




\section{Keywords}

Lambosacral, Lesion, Astrocytoma, Ependymoma

\section{Introduction}

The lumbosacral plexus consists of the ventral rami from the L2 to S3 nerve roots, with some additions from the L1 and S4 nerve roots as well [1]. It contains around 200000 axons and gives rise to six sensory nerves to the thigh and leg and six major sensorimotor nerves innervating about 43 muscles [2]. The lumbosacral plexus actually consists of two separate parts, the lumbar and the sacral plexus lying above and below the pelvic rim, respectively, which are connected by the so-called lumbosacral trunk. The lumbar part of the plexus lies embedded between and in the paraspinal quadratus lumborum and psoas muscles, making it vulnerable to local trauma or compression [3] [4]. On the other hand, the sacral plexus lies in the pelvis making it vulnerable to pelvic pathology. For diagnostic purposes it is important to make a clinical distinction between pathology in either the lumbar or sacral part of the plexus, or both, as the etiology and diagnostic approach (e.g., choice of muscles for needle EMG or MRI technique) varies accordingly [5].

Generally speaking the cause of a lumbar or sacral plexopathy is usually obvious or has only a limited differential diagnosis once the clinical diagnosis has been made. The lumbosacral plexus is most frequently affected by diabetic amyotrophy (a.k.a. Bruns-Garland syndrome or diabetic radiculoplexo-neuropathy), an acute onset and very painful disorder occurring in about $0.8 \%$ of all patients with diabetes mellitus [6]. Other less frequent causes are disruptive trauma of the pelvic ring or lumbar region, iatrogenic damage with surgery or radiation, and entrapment neuropathy of the lumbosacral trunk during the last trimester of pregnancy [7]. Compression by a tumor, hematoma in coagulopathy, aneurysm or abscess, and inflammatory, infectious or hereditary neuropathies are all rarer causes of a lumbosacral plexopathy. Lumbar degenerative spinal pathology and stenosis can also lead to compression of multiple nerve roots, which can cause pain, sensory symptoms, and paresis in a plexus-like distribution [8] [9]. Usually, the additional symptoms of lower back pain and provocation of symptoms by certain activities (e.g., walking) provide the clue to this diagnosis. Another mimic of a lumbosacral plexopathy can be lower-limb motor neuron disease, either idiopathic as in amyotrophic lateral sclerosis (ALS) or spinal muscular atrophy (SMA), or infection in endemic areas as in a poliomyelitis anterior acuta [10]. In young patients a previously undiscovered tethered spinal cord can also present with patchy paresis and sensory symptoms in the legs [11] [12].

The aim of this study is to evaluate outcome after surgical removal with duration of symptoms and nature of the lumbo-sacral spinal tumour. 


\section{Patients and Methods}

\subsection{Study Design and Patient Characteristics}

During the period between 2015 and 2017, thirty cases were studied and surgically managed in Al-Azhar University Hospital, Damietta. Cases were selected for surgery if they were diagnosed as having a symptomatizing lumbosacral lesion. All patients were subjected to a full history taking, full clinical examination, and routine laboratory investigations. Magnetic resonance imaging (MRI) of the spinal cord was done in all patients, while computed tomography (CT) was done in three cases, and spinal angiography was done in three cases and MRI brain and $\mathrm{CT}$ abdomen done when are needed. All of the thirty surgical cases were operated upon under general endotracheal intubation anesthesia. A posterior surgical approach was done in all cases. The operating surgical microscope was used in all cases. Patient's baseline characteristics are reported in Table 1.

\subsection{Surgical Procedures}

The operating surgical microscope was used in all cases. Surgical intervention was indicated by a combination of presenting symptoms (radiculopathy and/or myelopathy) and radiographic findings of magnetic resonance imaging (MRI), and computed tomographic (CT). The neuroimaging procedure of choice was contrast-enhanced MRI. All patients were operated upon under general endotracheal anesthesia. Pre-anesthetic intravenous medications were given on induction of anesthesia in the form of a 1 gram of 3rd generation cephalosporin, and $8 \mathrm{mg}$ decadrone. As mentioned before, 3 cases had intra-operative somatosensory evoked potential monitoring; the electrodes were positioned prior to placing the patient in the prone position.

All patients were operated upon in the prone position, using a spinal bridge, with flexion of the hips and knees. To prevent epidural venous congestion, complete freeing of the abdomen was done. The skin was prepared using the usual

Table 1. Shows the baseline patients characteristics.

\begin{tabular}{ccc}
\hline \multicolumn{2}{c}{ Patients Characteristics } & Mean \pm SD, N (\%) \\
\hline Age & Male & $38.96 \pm 15.42$ \\
Fender & Female & $15(50 \%)$ \\
& Ependymoma & $15(50 \%)$ \\
Astrocytoma & $8(27 \%)$ \\
Meningioma & $6(20 \%)$ \\
Nerve sheath tumor & $5(17 \%)$ \\
Hemangioblastomas & $5(17 \%)$ \\
Epidermoid & $3(10 \%)$ \\
Epidermoid of filum terminale & $1(3 \%)$ \\
& $2(6 \%)$ \\
\hline
\end{tabular}


protocol for surgical antisepsis (10\%, povidone iodine for 3 minutes). A midline skin incision was performed starting 2 levels above and below the desired site for laminectomy guided by fluoroscopy. This was followed by sub-periosteal muscle separation bilaterally. A laminectomy was performed for all of the cases (laminoplasty was do not done as there was no suitable tools), extending one level above and below the desired site for surgical exposure. A midline I-shaped dural incision was then done. Stay stitches of the dura to the adjacent muscles were done to keep the dura open. The operating microscope was brought in at this stage for all of the cases.

Using microsurgical technique, the blood vessels on the dorsal aspect of the spinal cord were mobilized from the midline using sharp arachnoid dissection, if they happen to cross the midline at the desired myelotomy site. A myelotomy was then performed in intramedullary lesions only. In cases where evoked potentials were available, the site of myelotomy was guided by the readings of the evoked potential. The tumor was then identified and its consistency, color, and vascularity were assessed. Internal debulking was done, taking care not to thin out the tumor too much in order not to lose tumor grip during dissection. The tumor was then dissected from the surrounding normal cord tissue using a combination of aspiration and a blunt dissection. The upper and lower pole of the tumor was then followed till a total or subtotal tumor resection was achieved.

\subsection{Outcomes Evaluation}

The study outcomes classified into 5 categories: 1) Excellent: those patients with a total surgical removal of the lesion, with no postoperative new neurological deficit; 2) Good: those patients with a subtotal surgical removal of the lesion, and no postoperative new neurological deficit; 3) Fair: those patients with a total surgical removal of the lesion, but with a postoperative neurological deficit; 4) Poor: those patients with a subtotal surgical removal of the lesion, and a postoperative neurological deficit; 5) Death.

\subsection{Statistical Analysis}

Categorical data were expressed as frequencies and percentages and compared using the Chi-Square test, while continuous data were expressed as means \pm standard deviations of the mean and compared using the student's $\mathrm{T}$ test of two independent samples. We performed multivariate logistic regression to predict the independent factors for motility. All analyses were conducted using the SPSS (version 22 for windows) and the significance of performed tests was assigned at $\mathrm{p}$ value $<0.05$

\section{Results}

\subsection{Demographic Data}

Thirty patients were included in the current study; the commonest lesion in this study was ependymoma eight patients (27\%), astrocytoma six patients (20\%), 
and meningioma five patients (17\%). Nerve sheath tumor comprised five cases (17\%) with the hemangioblastomas three cases (10\%), epidermoid one case (3\%), and ependymoma of the filum terminale two cases (6\%). The mean age of the included patients was $38.96 \pm 15.42$. The mean duration of lesions was 13.66 \pm 10.93 (Figures 1-5).

\subsection{Efficacy Outcomes}

Of all included patients, 14 cases (46.6\%) shows excellent status, 11 cases (36.6\%) good status, two cases (6.6\%) fair status, three cases (10\%) poor status, and we have no death cases.

\subsection{Relationship of Imaging Characteristics to Pathology}

We observed that it was extremely difficult to differentiate ependymoma and
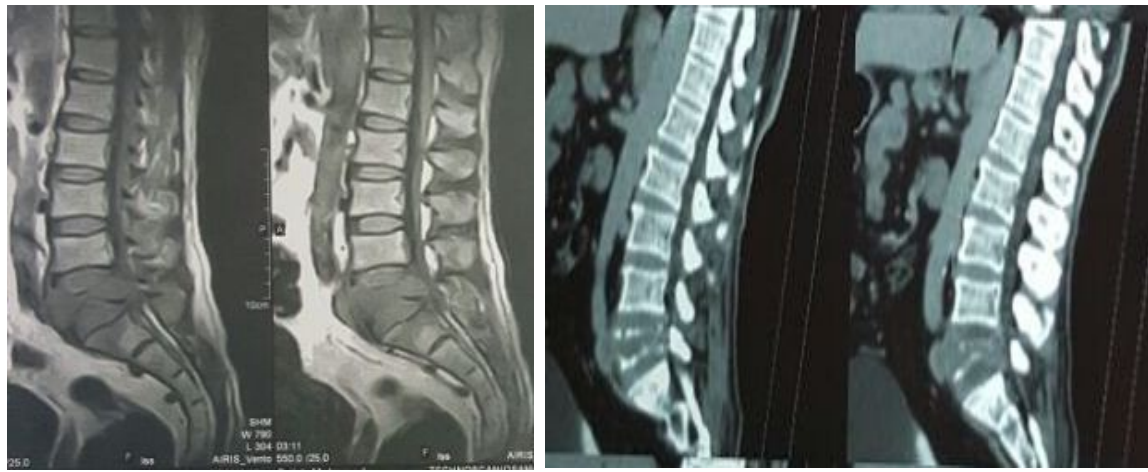

Figure 1. Preoperative MRI and CT for destructive lesion for L5 with significant cord compression.
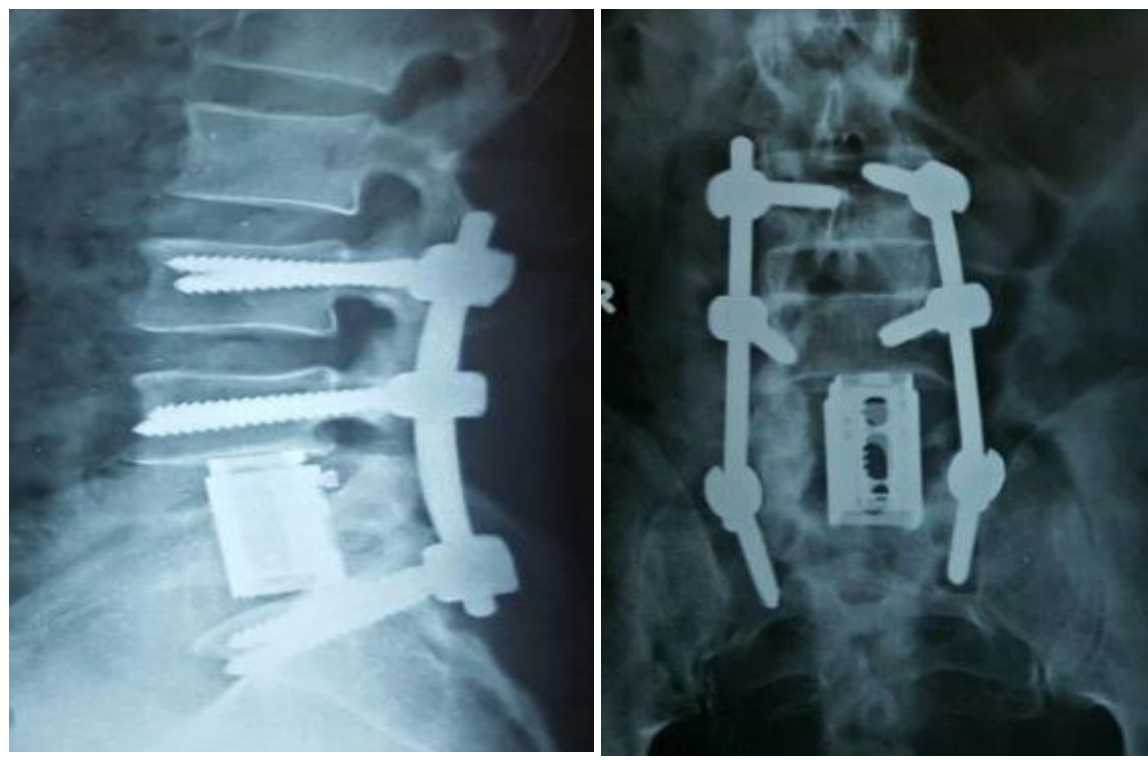

Figure 2. Postoperative $\mathrm{x}$ ray LSS shows posterior PLIF with transpedicular fixation after resection of the tumor involving $\mathrm{L} 5$. 

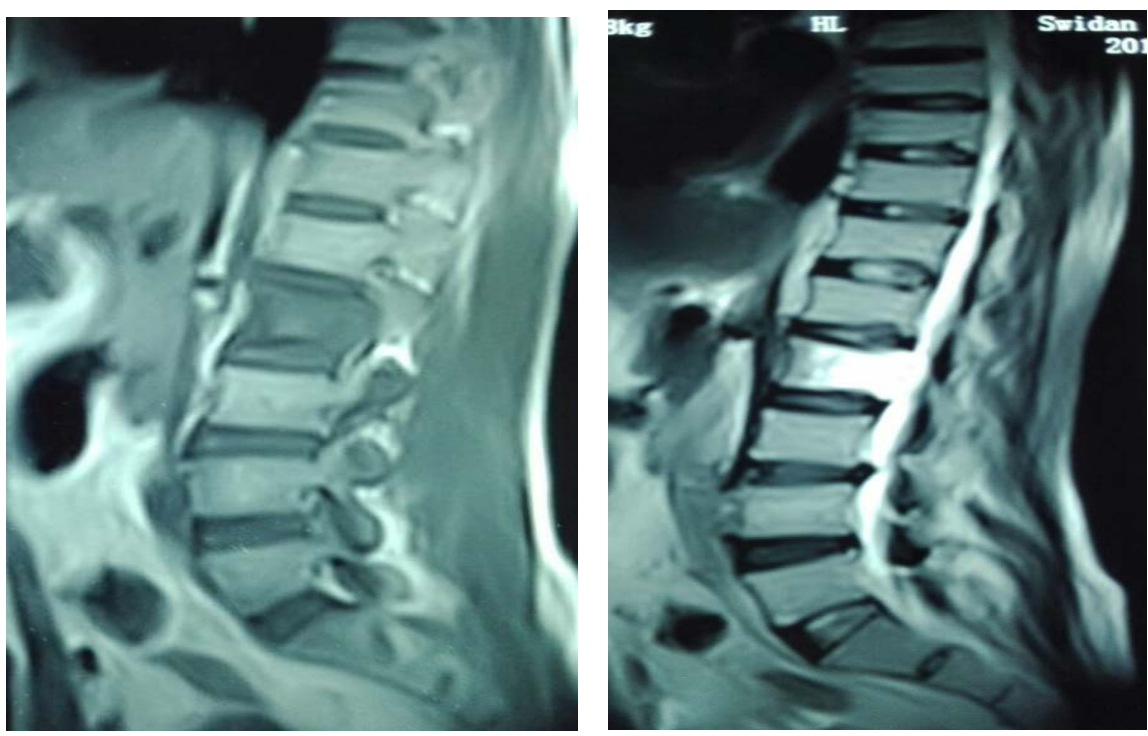

Figure 3. Preoperative MRI showed decrease height of the L2 vertebral body on the right side with large bony lesion displaying abnormal signal intensity.

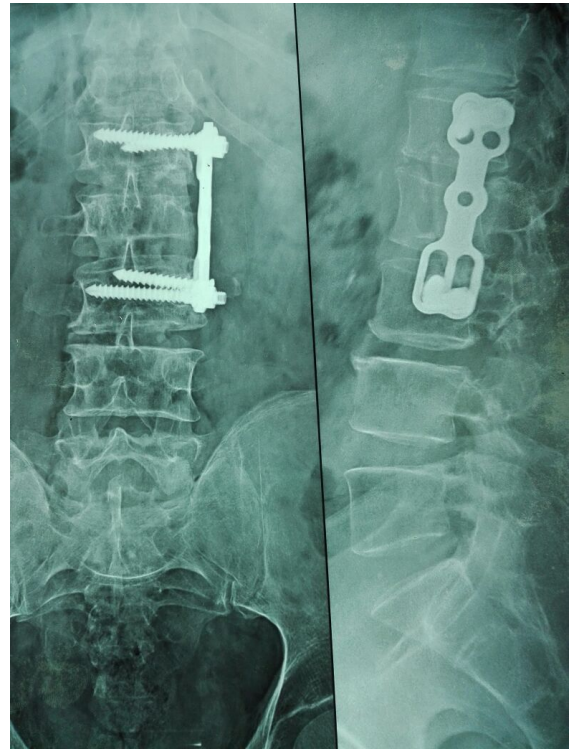

Figure 4. Through the aterolateral retroperitoneal approach an L2 subtotal corpectomy and total tumor excise with iliac bone graft and stabilization using plate and screws was performed.

astrocytoma on T1WI and T2WI basis. Post-contrast imaging proved that ependymomas tended to be more homogenously and intensely enhancing than astrocytomas. In addition, it helped in differentiating between syringomyelia and tumor-related cyst.

\subsection{Site in Relation to Pathology}

Regarding the site relation to pathology, our results demonstrated that 14 cases (47\%) in the lumbo-sacral cord, 10 cases (33\%) of tumors were located in the 


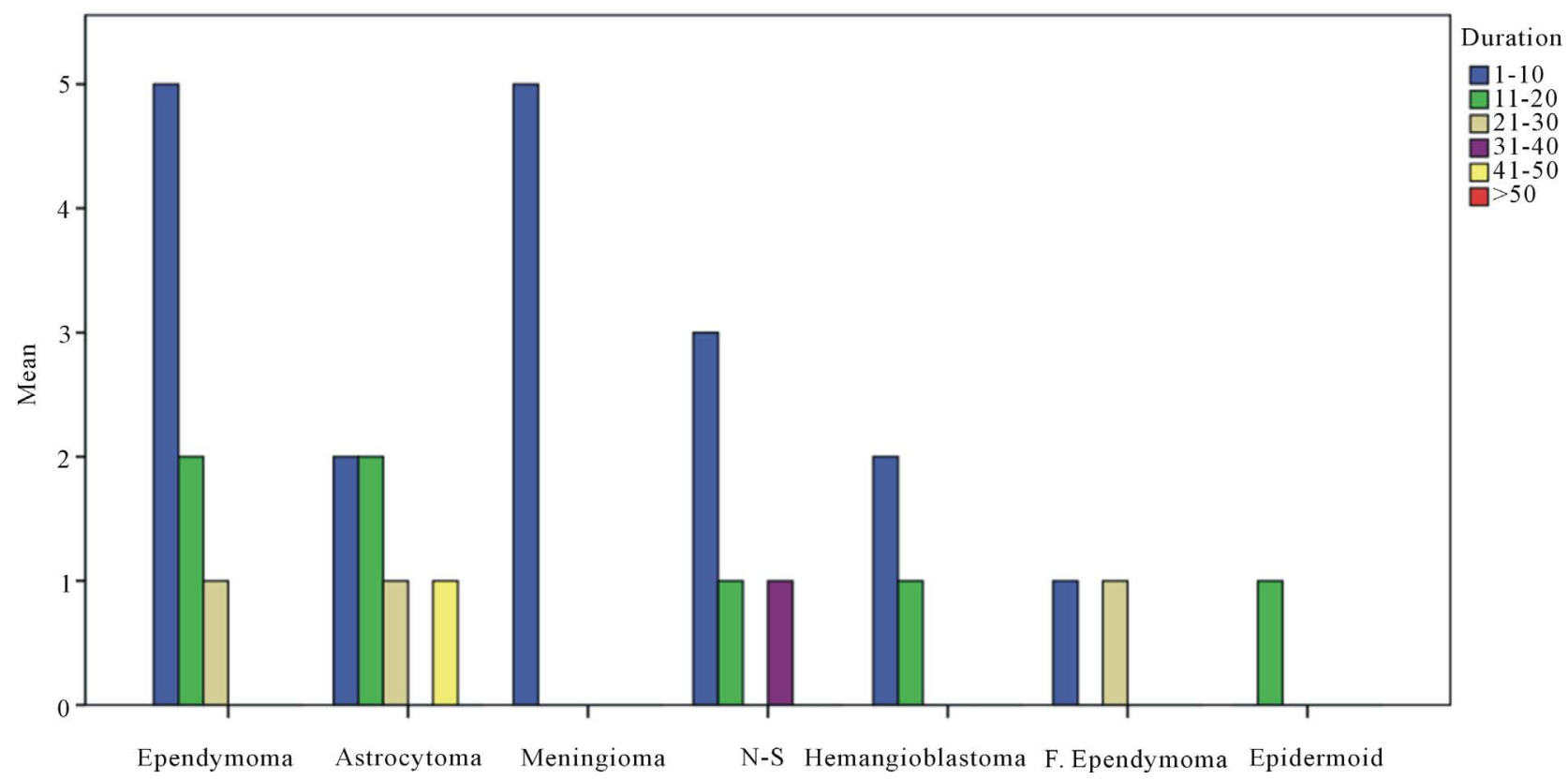

Figure 5. Shows the duration of each lesion type.

upper lumber cord, and three cases (10\%) of tumors were located in the lower lumber cord, and the remaining three cases (10\%) in the Sacro and sacro-coccygeal region.

\subsection{Treatment Modalities in Relation to Pathology}

Management lines of cases encountered in this study included medical treatment preoperatively, surgical decompression, radiotherapy, chemotherapy. But the choice of particular management plan was tailored for each case. Surgery was the primary treatment modality of choice in all cases. Cases were considered to be indicated for surgery if they had a symptomatizing intra-dural spinal cord mass lesion.

\subsection{Safety Outcomes}

Complications were encountered in eight cases (23\%) of patients. The commonest problem was a postoperative CSF leak. This complication is avoidable with a meticulous dura suture and tight soft-tissue closure, especially of the muscular layer. In recurrent cases, soft-tissue scarring puts the patient at an increased risk for leak. In such instances we recommend insertion of a lumbar drain at surgery and to keep it for about one week as a preventive measure, especially if a duroplasty has been inserted. Depending on the intraoperative situation, it may even be advisable in selected cases to insert a layer of fascia lata to reinforce the dura suture. There were three cases of CSF leak which treated conservatively and they improved. Other complications include wound infection two cases post operation.

\subsection{Outcomes in Relation to the Pathology}

Of the surgical cases, 19 cases (63\%) were totally resected, while 11 cases (37\%) 
were gross totally resected. The largest number of total resections in our study were meningiomas ( 5 cases out of the 5 cases, 100\%) and ependymomas ( 7 cases out of the 8 cases, $85 \%$ ), and nerve sheath tumor ( 4 cases out of 5 cases, 80\%) while the largest number of cases that were gross totally resected was astrocytomas ( 6 cases out of the 6 cases, 100\%). This indicates that spinal cord ependymomas, meningiomas and nerve sheath tumor are amenable to total resection, while achieving total resection in astrocytomas is not possible.

\section{Discussion}

This study concerned with 30 cases of lumbosacral tumor, the commonest lesion in this study was ependymomas 8 cases (27\%), followed by astrocytomas 6 cases (20\%), followed by meningiomas 5 cases (17\%). Nerve sheath tumor comprised 5 cases $(17 \%)$ with the hemangioblastomas 3 cases (10\%), epidermoid 1 case (3\%), and ependymoma of the filum terminale 2 cases (6\%). Fourteen cases (47\%) in the lumber cord, 10 cases $(33 \%)$ of tumors were located in the lumbo-sacral cord, 3 cases (10\%) of tumors were located in the sacrum cord, and the remaining 3 cases (10\%) in the sacro-coccygeal area. The youngest patient was at 10 years old and the oldest one was at 72 years old with average age 39 years. And there were 15 patients male and 15 patients female with equal percentage.

We found that while schwannomas affected younger male patients, meningiomas tended to occur in women and older patients. Clinically, the commonest presenting manifestations were sensory. These were followed by motor manifestations, followed by sphincteric troubles [13].

Regarding the sensory manifestations, the commonest was pain 23 cases (77\%). It presented in different modalities, spinal axial pain (being most severe over the affected region), radicular (either through compression of the nearby roots, or through direct invasion of the root) being common in the lumbosacral region. Pain is thought to be secondarily to dural distension, rather than infiltration of the spinothalamic tracts. Different modalities of sensory loss are including sensory level, hemi-hypothesis, saddle sensory loss, and central cord syndrome.

The commonest in this study was sensory level 7 cases (23\%); and hemi-hypothesis 6 cases (26\%), followed by central cord syndrome 7 cases (23\%). The possible vague sensory symptoms as well as the slow growth rate of tumors, the ability of the spinal cord to tolerate slowly generated compression are responsible for failure of early diagnosis of benign intramedullary tumors. In addition, the common resemblance of the sensory manifestations of the intra-dural tumors with other pathologies, such as inflammatory or disc prolapse, may cause mis-diagnosis and hence delay in initiating the proper management.

As for the commonest motor manifestation, monoparesis was present in 12 cases (40\%); followed by paraparesis 7 cases (23\%). 16 patients $(53 \%)$ complained of sphincteric disturbances. The commonest of which was precipitancy 8 cases (26\%); followed by incontinence 6 cases $(20 \%)$ and retention 2 cases $(6 \%)$ 
and baseline urodynamics studies was done for them. Spinal deformity may be the presenting symptom especially in the pediatric age group, or present in association of an intramedullary tumor.

The duration of symptomatology ranged from 1 month to 60 months, with an average of 13.6 months. Almost more than half of the patients 18 cases (60\%) presented within 10 months of the onset of symptomatology. Only 5 cases presented after 20 months. The relatively short period of symptomatology was probably due to the relative ease and accuracy of modern imaging studies, mainly MRI, when compared to the past. Schwannomas had a statistically longer duration of symptoms (15.6 months) than did meningiomas (6 months,) for cervical and thoracic tumors.

Regarding the relationship of imaging characteristics to pathology, we observed that it was extremely difficult to differentiate ependymomas and astrocytomas on T1WI and T2WI basis. Post-contrast imaging proved that ependymomas tended to be more homogenously and intensely enhancing than astrocytomas. In addition, it helped in differentiating between syringomyelia and tumor-related cyst. Klekamp and Samii, in their study of 100 intramedullary cases found that, the presence of an associated syrinx favored the respectability of the rumor, because it indicated a displacing rather than an infiltrating tumor. Patients with syringomyelia tended to recover from surgery sooner [14].

Unfortunately, no laminoplasty was done in our cases as there were no suitable tools for that so all of our cases laminectomy was the only procedure. Furthermore, atrophy and abnormal innervation of neck and back muscles may cause muscular imbalances, which alone may be sufficient to induce spinal instability despite reinserted laminae.

Furthermore, spinal deformities may be present before surgery, as demonstrated earlier. Nevertheless, we recommend reinsertion of the laminae in all patients with miniplates after removal of the tumor, to restore the anatomy as far as possible. Avoiding fixation of the neck and back muscles to an epidural scar, one may consider a favorable effect to avoid neck and back pain related to muscular tension on the dura. Reinserted laminae also make a reoperation easier in the case of a tumor recurrence. We believe that these are reasons enough, even if a postoperative deformity cannot be prevented in all cases and monitoring by experienced orthopedists is still recommended, and mandatory especially in children.

The major factor determining permanent morbidity, however, is the preoperative status of the patient. Numerous studies emphasized that the major factor determining long-term outcome is the preoperative neurological status. This can be attributed to marked differences in surgical morbidity in relation to the preoperative status. For patients with a preoperative Karnofsky score above 70 (i.e., patients able to live independently without assistance), permanent morbidity was $9 \%$. For patients with scores between 40 and 70 the figure rose to $18 \%$, and for patients with scores below 40, surgical morbidity was $30 \%$. Moreover, a 
new postoperative deficit had considerably more serious functional consequences for those patients who were already significantly disabled preoperatively [15] [16]. The small sample and un-ability to make randomization are considered as limitations in our study.

\section{Conclusion}

According our finding, we conclude that excellent outcome was associated with ependymoma followed by meningioma, nerve sheath tumors and extramedullary ependymoma of filum terminale. Accurate and rapid diagnosis prior to surgery is also associated with good outcome. The presence of new sophisticated utilization namely, MRI, operating microscope, and intraoperative somato-sensory evoked potential, all have added to armaments of the surgeon to provide a better results. This is evident when the recent results compared to the past.

\section{References}

[1] Adams, M.A., Freeman, B.J., Morrison, H.P., Nelson, I.W. and Dolan, P. (2000) Mechanical Initiation of Intervertebral Disc Degeneration. Spine, 25, 1625-1636. http://www.ncbi.nlm.nih.gov/pubmed/10870137 https://doi.org/10.1097/00007632-200007010-00005

[2] Albrecht, S., Crutchfield, J.S. and SeGall, G.K. (1992) On Spinal Osteochondromas. Journal of Neurosurgery, 77, 247-252. http://www.ncbi.nlm.nih.gov/pubmed/1625013 https://doi.org/10.3171/jns.1992.77.2.0247

[3] Aronson, D.D., Kahn, R.H., Canady, A., Bollinger, R.O. and Towbin, R. (1991) Instability of the Cervical Spine after Decompression in Patients Who Have Arnold-Chiari Malformation. Journal of Bone \& Joint Surgery, 73, 898-906.

http://www.ncbi.nlm.nih.gov/pubmed/2071622 https://doi.org/10.2106/00004623-199173060-00012

[4] Bach, F., Larsen, B.H., Rohde, K., Børgesen, S.E., Gjerris, F., Bøge-Rasmussen, T., et al. (1990) Metastatic Spinal Cord Compression. Occurrence, Symptoms, Clinical Presentations and Prognosis in 398 Patients with Spinal Cord Compression. Acta Neurochirurgica, 107, 37-43.

http://www.ncbi.nlm.nih.gov/pubmed/2096606 https://doi.org/10.1007/BF01402610

[5] Cohen-Gadol, A.A., Zikel, O.M., Koch, C.A., Scheithauer, B.W. and Krauss, W.E. (2003) Spinal Meningiomas in Patients Younger than 50 Years of Age: A 21-Year Experience. Journal of Neurosurgery, 98, 258-263. http://www.ncbi.nlm.nih.gov/pubmed/12691381

[6] Coloma-Valverde, G., Granado-Peña, J.M. and Estévez-Rosas, S. (1999) C6-C7 Subluxation and the Importance of Simple Radiology in the Emergency Department. A Case Report and Literature Review. Revista De Neurologia, 29, 1035-1037. http://www.ncbi.nlm.nih.gov/pubmed/10637866

[7] Dommisse, G.F. (1974) The Blood Supply of the Spinal Cord. A Critical Vascular Zone in Spinal Surgery. Journal of Bone and Joint Surgery, 56, 225-235. http://www.ncbi.nlm.nih.gov/pubmed/4854669 https://doi.org/10.1302/0301-620X.56B2.225

[8] El-Mahdi, M.A., Abdel Latif, F.Y. and Janko, M. (1981) The Spinal Nerve Root "In- 
nervation", and a New Concept of the Clinicopathological Interrelations in Back Pain and Sciatica. Neurochirurgia, 24, 137-141.

http://www.ncbi.nlm.nih.gov/pubmed/6211633

[9] Lindahl, O. (1966) Hyperalgesia of the Lumbar Nerve Roots in Sciatica. Acta Orthopaedica Scandinavica, 37, 367-374.

http://www.ncbi.nlm.nih.gov/pubmed/5961766

https://doi.org/10.3109/17453676608989427

[10] Oegema, T.R. (1993) Biochemistry of the Intervertebral Disc. Clinics in Sports Medicine, 12, 419-439. http://www.ncbi.nlm.nih.gov/pubmed/8364983

[11] Epstein, F. and Epstein, N. (1982) Surgical Treatment of Spinal Cord Astrocytomas of Childhood. A Series of 19 Patients. Journal of Neurosurgery, 57, 685-689.

http://www.ncbi.nlm.nih.gov/pubmed/7131070

https://doi.org/10.3171/jns.1982.57.5.0685

[12] Myles, L.M., Gupta, N., Armstrong, D. and Rutka, J.T. (1999) Multiple Extradural Arachnoid Cysts as a Cause of Spinal Cord Compression in a Child. Case Report. Journal of Neurosurgery, 91, 116-120. http://www.ncbi.nlm.nih.gov/pubmed/10419356

[13] Constantini, S., Lidar, Z., Segal, D. and Corn, A. (2012) Delay in Diagnosis of Primary Intradural Spinal Cord Tumors. Surgical Neurology International, 3. 52. http://www.surgicalneurologyint.com/text.asp?2012/3/1/52/96075

[14] Samii, M. and Klekamp, J. (1994) Surgical Results of 100 Intramedullary Tumors in Relation to Accompanying Syringomyelia. Neurosurgery, 35, 865-873

http://www.ncbi.nlm.nih.gov/pubmed/7838335 https://doi.org/10.1227/00006123-199411000-00010

[15] Foo, D. and Rossier, A.B. (1981) Preoperative Neurological Status in Predicting Surgical Outcome of Spinal Epidural Hematomas. Surgical Neurology, 15, 389-401. http://www.ncbi.nlm.nih.gov/pubmed/9760981 https://doi.org/10.1016/0090-3019(81)90178-6

[16] Halaszynski, T. (2013) Influences of the Aging Process on Acute Perioperative Pain Management in Elderly and Cognitively Impaired Patients. The Ochsner Journal, 13, 228-247.

http://www.pubmedcentral.nih.gov/articlerender.fcgi?artid=3684333\&tool=pmcent $\underline{\text { rez\&rendertype }=\text { abstract }}$ 